\title{
Reliability of Visual Inspection After Acetic Acid Staining in Screening for Cervical Pre-malignant Lesion Among Female Subjects in a Rural Tertiary Hospital in Nigeria
}

\author{
Gabriel Olusegun Emmanuel ${ }^{1}$, Ajetunmobi Oluwaserimi Adewumi ${ }^{1}$, \\ Akomolafe Akinwumi Oluwole $^{2}$, Bushari Olusegun Adesola ${ }^{1}$, Adeniyi Adebayo Augustine ${ }^{3}$, \\ Dada Samuel Ayokunle ${ }^{4}$
}

${ }^{1}$ Department of Family Medicine, Federal Teaching Hospital Ido-Ekiti, EkitiState, Nigeria

${ }^{2}$ Department of Morbid Anatomy, Obafemi Awolowo University Ile-Ife, Osun State, Nigeria

${ }^{3}$ Department of Obstetrics and Gynaecology, Federal Teaching Hospital Ido-Ekiti, Ekiti State, Nigeria

${ }^{4}$ Department of Internal Medicine, Renal Unit, Ekiti State University Teaching Hospital, Ado-Ekiti, Nigeria

\section{Email address:}

layodeg@gmail.com (G. O. Emmanuel)

\section{To cite this article:}

Gabriel Olusegun Emmanuel, Ajetunmobi Oluwaserimi Adewumi, Akomolafe Akinwumi Oluwole, Bushari Olusegun Adesola, Adeniyi Adebayo Augustine, Dada Samuel Ayokunle. Reliability of Visual Inspection After Acetic Acid Staining in Screening for Cervical Premalignant Lesion Among Female Subjects in a Rural Tertiary Hospital in Nigeria. Cancer Research Journal. Vol. 4, No. 1, 2016 , pp. 1-8. doi: $10.11648 /$ j.crj.20160401.11

\begin{abstract}
This study was carried out in order to assess the sensitivity and specificity of visual inspection after acetic acid staining (VIA) using Papanicolaou smear as the gold standard screening method for cervical premalignant lesion. Two hundred and twenty (220) consecutive and consenting women ages 20 - 70 years, attending Federal Medical Centre, Ido-Ekiti, Ekiti State, were recruited. Relevant data were collected using interviewer-administered semi-structured questionnaires. In conclusion, the prevalence of pre-malignant lesion of cervical cancer is high in this study. It is therefore recommended that awareness of cervical cancer and its screening should be improved through health education campaigns. VIA can serve as an alternative to Papanicolaou smear in resource-limited countries, since it has high level of specificity and sensitivity as Papanicolaou smear.
\end{abstract}

Keywords: Cervical Cancer, VIA, Pap Smear, Screening, Developing Countries

\section{Introduction}

Cervical cancer has remained a major health problem especially in the developing world despite the known preinvasive stage of this malignant condition and implementation of cervical cancer screening programs.[1,2] The developing countries accounted for 370,000 out of 466,000 cases of cervical cancer that were estimated to have occurred in the world in the year 2000. [3] This had increased to 493,000 cases worldwide in 2002 of which more than $80 \%$ of these cases occurred in the developing countries. [4]

Cervical cancer is an important public health problem among adult women in developing countries in South and Central America, Sub-Saharan Africa and South-East Asia. In these regions, it is either the most common or second most common cancer among women. About 274,000 or more deaths occur annually due to cancer of the cervix worldwide, and more than $80 \%$ of these deaths occur in developing countries.[5,6] Moreover, resource-poor areas, such as SubSaharan Africa, are currently witnessing a growing number of women infected with human immunodeficiency virus (HIV). This may further compound the problem because they have an increased risk of Human Papilloma Virus (HPV) infection, the causal agent of cervical cancer. [7]

The incidence of cervical cancer in sub-Saharan Africa is among the highest worldwide, with the available agestandardized rates ranging from 19.9 per 100,000 in Ibadan, Nigeria, through 35.7 per 100,000 in Bamako, Mali, to 41.7 per 100,000 in Kyadondo, Uganda.[8-11]

While evidence of effective screening programmes can be seen throughout the developed world, the burden and impact of the disease remains high in developing countries, where 
$85 \%$ of the disease-related deaths occur. $[12,13]$ The highest incidence has been observed in sub-Saharan Africa, Latin America, the Caribbean, South central Asia, and Southeast Asia. [14] In a study from University of Ilorin Teaching Hospital in Nigeria, Carcinoma of the cervix accounted for $63.1 \%$ of histologically confirmed gynaecological cancers over a period of ten years.[15] Similarly, a retrospective review study from University of Jos Teaching Hospital, Nigeria, identified cervical cancer as the commonest diagnosed between 1985 -1994. It took the second position during the subsequent ten years while breast cancer took the lead. In the study cervical cancer accounted for $14.7 \%$ in the first ten years but increased to $17.3 \%$ in the following ten years of the study. [16] All these suggest increase in cervical cancer burden in Nigeria.

Though cervical cancer awareness is generally low worldwide, it is worse in developing countries despite the increased prevalence of the disease in these countries. [17] To improve the level of awareness of cervical cancer and its screening coverage, there is the need to employ primary care approach. This will help to identify and proffer solutions to barriers to cervical cancer control. [18] The Primary Care Physician can achieve this through information and education given to members of the family. Research on families and health demonstrates that the family strongly influences most health behaviours, and that a family-oriented approach is the most effective and efficient way to prevent disease and promote health. [19]

The success of any screening is directly related to the percentage of the target population who are screened and the reliability of the screening test. The Physicians at the primary care levels have the opportunity and responsibility to influence both of these factors. [20] Primary care Physicians need to make preventive care a priority. When patients attend clinic for any reason, the physicians need to encourage all women qualified for screening to undergo screening tests, especially those who have never been screened. This requires effective communication of the purpose of screening, the mechanics of the test as well as available treatment modality. [19-21]

The difficulty in ensuring high-quality cytology-based services in many settings has led to significant interest in new approaches to screening for precancerous lesions. Of these, visual inspection of the cervix is a promising option; especially for low-resource settings. [22]

Visual inspection after acetic acid staining (VIA) is defined as visual inspection of the acetic acid-swabbed cervix without any magnification. Results of several studies in developing countries suggest that VIA is as sensitive as Pap smears in detecting high-grade dysplasia in many settings, although not as specific. [22, 23]

VIA is a relatively simple and of low-technology approach that is minimally reliant upon infrastructure for performance. Non-physicians can perform the procedure, provided that they receive adequate and continuing training. Furthermore, results of the procedure are available immediately; making it possible, in principle, to provide treatment during the same visit. Therefore, VIA may be a very important procedure to use at primary and secondary health care level to.[22, 23] screen for cervical cancer.

\section{Objective}

To determine the sensitivity and specificity (validity) of VIA using Papanicolaou smear as gold standard in criterion validation.

\section{Method of Study}

This is a descriptive, cross-sectional hospital-based study of validity of visual inspection after acetic acid application for cervical cancer screening. Papanicolaou smear was used as the gold standard for cervical cancer screening. It was conducted in the General Outpatient clinic and Gynaecology Clinic of Federal Teaching Hospital (FTH) Ido-Ekiti, Ekiti State, Nigeria. The target population for this study consisted of sexually active women ages 20 to 65 years who attended the clinics during the period of the study.

Federal Teaching Hospital (FTH) Ido-Ekiti is situated in Ekiti state in the Western part of Nigeria. The Hospital serves as a referral centre for the specialist Hospitals, general Hospitals, and comprehensive health centres in its environs, though it is sited in the rural settlement of the state about 45 kilometers to the capital city.

Inclusion Criteria are:

i. Women of age 20 years to 64 years who have been sexually active for at least two years irrespective of their Human Immunodeficiency Virus status.

ii. Women aged 65 to 70 years who had never had a cervical cancer screening test after their 50th birthday.

iii. Women ages 25 years and above who had never been expose to sex but were willing to participate.

Exclusion criteria are:

i. Women with previous history of hysterectomy with cervix completely removed.

ii. Women with previous history of cervical cancer.

iii. Women who were menstruating (sample was not taken during menstrual flow, such patients were asked to come back few days after completing their menstruation).

iv. Women who have had Pap's smear within the last two years at the time of the study

v. Women with obvious genital infection e.g. obvious vagina discharge.

vi. Women who had used vagina cream or pessaries or douching within 24 hours (were given appointment for the test).

vii. Women who were pregnant (for medicolegal reasons).

Note: Women with obvious genital infections were managed and were asked to come back when the infection resolved.

The calculated sample size was 220 and the selection of 
the samples from the two clinics was based on the mean attendance of women of age 20-65years in the two months preceding the month of commencement of the data collection (February and March, 2009 as reported earlier).Each sample was calculated thus; the average attendance per clinic divided by the total attendance in the two clinics multiplied by the desired sample size. For example, in the General Outpatient Department of Federal Teaching Hospital, Ido-Ekiti, the sample selected and taken was calculated as 460/610 × $220=$ 166.In the same manner in the Gynaecology Clinic, fifty four $(150 / 610 \times 220=54)$ women were screened. This was done to ensure equal distribution.

\subsection{Ethical Clearance and Consent}

Ethical clearance was obtained from the Ethical and Research Committee of FTH Ido-Ekiti (Appendix 1). Informed written consent was obtained from each subject.

\subsection{Material}

Disposable plastic Cusco speculums were used; the swabs on sticks used were made by the researcher and autoclaved in the operating theatre of the Hospital. Other materials used were adequate light source, frosted-end glass slides, HB pencil, alcohol fixative, latex glove, Ayre's wooden spatula, coupling rack, acetic acid (vinegar). Phone calls were made to inform the subjects to come for their cytology results.

The estimated cost of the materials common to both procedures (VIA and Pap smear) was $\$ 27,900.00$. The acetic acid that was used cost 1000.00 and the unit cost of VIA per patient was $\$ 200.00$ (a total of $\$ 44,000.00$ for 220 subjects). The estimated cost of the Papanicolaou smear, including phone calls, slides, and Ayre's spatula specific to Papanicolaou smear was $\$ 245,100.00$ (Cervical cytology per subject was 1000.00). The cost of transport of the subjects back for the results of Papanicolaou smear is not included, it is unknown. Hence the total cost for VIA screening test for 220 participants was $\$ 72,900.00$, and that of Papanicolaou test for 220 participants was $\$ 273,000.00$. The aforementioned total costs of the two test procedures were borne by the researchers. Cost of the transport back to collect the result of the Pap smear was borne by each participant.

\subsection{Method of Data Collection}

The women waiting to be seen for different health matters in the earlier mentioned outpatient clinics were informed that two screening tests for the same purpose (but not HIV screening) were available. They were also informed that the tests were free and they did not need to pay any amount of money as related to the two tests. The women were told that they would be interviewed with questionnaires and thereafter they would be educated on the purpose and modality of the screening tests. They were also assured that the screening tests would not, by any means be harmful, rather, it would be beneficial to them. The patients were also informed that they were free to withdraw from the study at any point if they so wish.

The questionnaires were administered to those who signified interest and thereafter health education talk was given on cervical cancer and its screening to each woman by the investigators. The women were made to understand what cervical cancer was, the risk factors and the available preventive methods (screening). They were also informed on how the screening tests would be conducted. The health education was not only given in English language, it was also given in local language (Yoruba) and Pidgin English depending on what the subject understood best. After the health talk, those who indicated interest in the screening and who met the inclusion criteria had the screening tests done.

Participants were interviewed by a trained female midwife and the researchers who used a pre-tested semistructured questionnaire. This questionnaire assessed their socio-demographic data, level of awareness, and possible risk factors among other things. The questionnaire was developed by the researchers based on extensive literature review and tailored towards the objectives of the study.

The study objectives were explained, verbal and informed written consents were obtained from the participants. Immediately after the participant's history was taken with the questionnaire, both screening tests were done for the consenting women. Each woman was placed in a lithotomic position on a screened examination couch with a female chaperone in attendance. Non-lubricated sterilized plastic bivalve speculum was inserted into the vagina and a cervical cytology specimen was obtained with a wooden Ayres spatula. The wooden Ayres spatula was rotated through 360 degree around the transformation zone to scrape the cervix around the entire transformation zone to obtain an adequate specimen. Immediately after a cytology specimen was obtained, the investigators cleaned away any excess mucus thoroughly with a sterile water-soaked swab, and applied a solution of $4 \%$ acetic acid to the cervix with a cotton-tipped applicator and waited for one minute. With the aid of a handheld flashlight, investigators then visually inspected the whole cervix. Categories of VIA findings were recorded on the study questionnaire.

The three of the researchers who had training and experience on VIA observed the cervix one after the other and recorded their findings separately. This was done at the same time in all the subjects used in the two clinics in order avoid inter or intra-observer error.

The cervical cytology specimen taken was immediately smeared on four glass slides per specimen. Four glass slides were used so as to increase the yield of the cervical cells. The smeared glass slides were carefully arranged in a coupling rack and dipped in a basin containing $95 \%$ alcohol to fix them.

The glass slides were well labeled; the four glass slides for a specimen carried the same serial number as the serial number on the client questionnaire and cytology request form for that specimen. It had been confirmed that such serial number was not being used for any other cytology sample in the laboratory where the samples were analyzed. The woman's name, serial number, age, tribe and relevant clinical history were recorded on the cytology forms. All the Papanicolaou specimens were processed and analyzed in the 
Histopathology laboratory of FTH, Ido-Ekiti.

Patients were informed of the results of VIA at the end of the procedure and were contacted through their phone numbers when the results of Pap smear were ready. Patients that were found positive for the pre-cancerous lesion by either of the two procedures or both were referred to the Gynaecologist for further management. They were also informed of the benefit of cryotherapy treatment modality that was made available at a Comprehensive Health Centre by the State Government. Some of the patients afforded themselves the opportunity of the cryotherapy. The patients were advised to repeat VIA or Papanicolaou smear after six months of Post cryotherapy treatment.

In this study Papanicolaou smear was used as the gold standard for screening of cervical cancer and since there was no opportunity to do cone biopsy for histology (the diagnostic test) of the studied subjects. VIA was compared with Papanicolaou smear (another screening test), to determine the criterion or concurrent validity of the VIA.

\subsection{Data Analysis}

All data collected were fed into the computer and analyzed using the Statistical Package for Social Sciences (SPSS) for windows software version 16.0. Summary statistics such as mean, mode, median, standard deviation, proportion and percentage were determined as applicable. The mean and standard deviation were calculated for numerical data (discrete variables) while ratios and proportions were calculated for categorical variables. Association between dependent and independent variables were compared using the Pearson's Chi square $\left(\chi^{2}\right)$ test and Fisher's exact test as applicable. The level of statistical significance was taken as $p$ values of equal or less than $0.05(\mathrm{p} \leq 0.05)$. The validity of VIA was determined by calculating the sensitivity, specificity, positive predictive value and negative predictive value of VIA using Papanicolaou smear as the gold standard. The prevalence of abnormal cytology smear was also calculated. These were calculated thus;

- Sensitivity $=$ True positive VIA / all with abnormal Pap smear.

- Specificity $=$ True negative VIA / all with normal Pap smear.

- Positive predictive value $=$ True positive VIA/ all positive VIA.

- Negative predictive value $=$ True negative VIA / all Negative VIA.

- Prevalence of abnormal cervical finding = $\frac{\text { True positive VIA + false negative VIA }}{\text { Sample size }}$.

The measure of agreement between VIA and Papanicolaou smear tests was also determined by Cohen's kappa test using the statistical package for social science. Cohen's Kappa measures the agreement between the evaluations of two raters when both are rating the same object. A value of one indicates perfect agreement and a value of 0 indicates that the agreement is not better than chance. [24] The interpretation of kappa result is as thus; [25]
- Poor agreement $=$ less than 0.2.

- Fair agreement $=0.20$ to 0.39 .

- Moderate agreement $=0.40$ to 0.59 .

- Good agreement $=0.60$ to 0.79 .

- Very good agreement $=0.80$ to 1.0 .

Test of association is given in two decimal places and pvalue in three decimal places. All other values were rounded up to first decimal place

\section{Results}

Meanage \pm standard deviation of the respondents was 41.2 \pm 12.6 years, median age was 40 years (The age range was 20 to 70 years). Bimodal age occurred in the distribution and these were 30 and 33 years. 25th percentile of the age distribution was 31 years, 50 th percentile was 40 years while 75 th percentile was 50 years.

Table 1. Age group distribution of the respondents $N=215$.

\begin{tabular}{lll}
\hline Age group (years) & frequency & percentage \\
\hline $20-29$ & 42 & 19.5 \\
$30-39$ & 63 & 29.3 \\
$40-49$ & 53 & 24.7 \\
$50-59$ & 36 & 16.7 \\
$60-69$ & 13 & 6.0 \\
$70-79$ & 8 & 3.7 \\
Total & 215 & 100 \\
\hline
\end{tabular}

Table 2 below shows that majority of the studied subjects 209(97.2\%) belonged to the Christian faith. Slightly less than half, $98(45.6 \%)$, of them had tertiary education and only 10 $(4.7 \%)$ of them had no formal education. Majority of the subjects were Yoruba speaking. Petty traders, labourers, messengers (Hospital ward maids), farmers and other similar grades were the highest participants, 102 (47.5\%) (class iv).

Table 2. Other socio-demographic characteristics of the respondents. $N=215$.

\begin{tabular}{ll}
\hline Characteristics & N (\%) \\
\hline Religion & $209(97.2)$ \\
Christianity & $6(2.8)$ \\
Islam & \\
Educational & $98(45.6)$ \\
status education & $73(34.0)$ \\
Tertiary education & $34(15.8)$ \\
Secondary education & $10(4.7)$ \\
Primary formal & \\
No & \\
Occupation* & $14(6.5)$ \\
I & $43(20.0)$ \\
II & $34(15.8)$ \\
III & $102(47.5)$ \\
IV & $22(10.2)$ \\
V & \\
Ethnic group & $205(95.3)$ \\
Yoruba & $8(3.7)$ \\
Ibo & $2(1.0)$ \\
Hausa & \\
Others & \\
\hline
\end{tabular}

* = occupation as classified by Oyedeji. 
Table 3 shows that abnormal cytology was found with increasing age (especially LGSIL and HGSIL). Acute cervicitis was highest among age group 30-39 years. It is commoner below 49 years $(28.8 \%)$ when compare to age 50 years and above (18.1\%). Atypical Squamous cell of undetermined significance (ASCUS) was highest in age group 20-29 years (7.1\%). High grade squamous intraepithelial lesion (HGSIL) was seen from 40 years of age, highest at age group 50-59 years (13.9\%). Half of the 70-year old women (50\%) had LGSIL and one $(12.5 \%)$ of them had HGSIL.

From this Table, $16(10.1 \%)$ of the women in the reproductive age group (20-49 years) had LGSIL while $13(22.8 \%)$ of the women at age 50 to 70 years had LGSIL. One

$(0.6 \%)$ of the women in the reproductive age (20-49 years) had HGSIL while $7(12.3 \%)$ of the women in age 50 to 70 years had HGSIL.

Table 3. The cytology results.

\begin{tabular}{|c|c|c|c|c|c|c|}
\hline Age group & Normal & Acute cervicitis & ASCUS & LGSIL & HGSIL & Total \\
\hline & n (\%) & n (\%) & n (\%) & n (\%) & n (\%) & \\
\hline $20-29$ & $37(88.1 \%)$ & 0 & $3(7.1 \%)$ & $2(4.2 \%)$ & 0 & 42 \\
\hline $30-39$ & $44(69.8 \%)$ & $11(17.5 \%)$ & $1(1.6 \%)$ & $7(11.1 \%)$ & 0 & 63 \\
\hline $40-49$ & $37(69.8 \%)$ & $6(11.3 \%)$ & $2(3.8 \%)$ & $7(13.2 \%)$ & $1(1.9 \%)$ & 53 \\
\hline $50-59$ & $23(63.9 \%)$ & $2(5.6 \%)$ & $1(2.8 \%)$ & $5(13.9 \%)$ & $5(13.9 \%)$ & 36 \\
\hline $60-69$ & $8(61.5 \%)$ & 0 & 0 & $4(30.7 \%)$ & $1(7.7 \%)$ & 13 \\
\hline $70-79$ & $2(25.0 \%)$ & $1(12.5 \%)$ & 0 & $4(50.0 \%)$ & $1(12.5 \%)$ & 8 \\
\hline
\end{tabular}

Table 4 shows that the proportion of women with positive results of VIA increased with age. The positive result was highest among women in the age group $50-59$ years. This is the age group at which the highest proportion of HGSIL was seen in Table 3.

Table 4. The results of VIA $(N=215)$.

\begin{tabular}{lll}
\hline & Positive(N=43) & Negative (N=172) \\
\hline Age group & n (\%) & n (\%) \\
\hline $20-29$ & $1(2.4 \%)$ & $41(97.6 \%)$ \\
$30-29$ & $11(17.5 \%)$ & $52(82.5 \%)$ \\
$40-29$ & $13(24.5 \%)$ & $40(75.5 \%)$ \\
$50-29$ & $13(36.1 \%)$ & $23(63.9 \%)$ \\
$60-29$ & $4(30.8 \%)$ & $9(69.2 \%)$ \\
$70-29$ & $1(12.5 \%)$ & $7(87.5 \%)$ \\
\hline
\end{tabular}

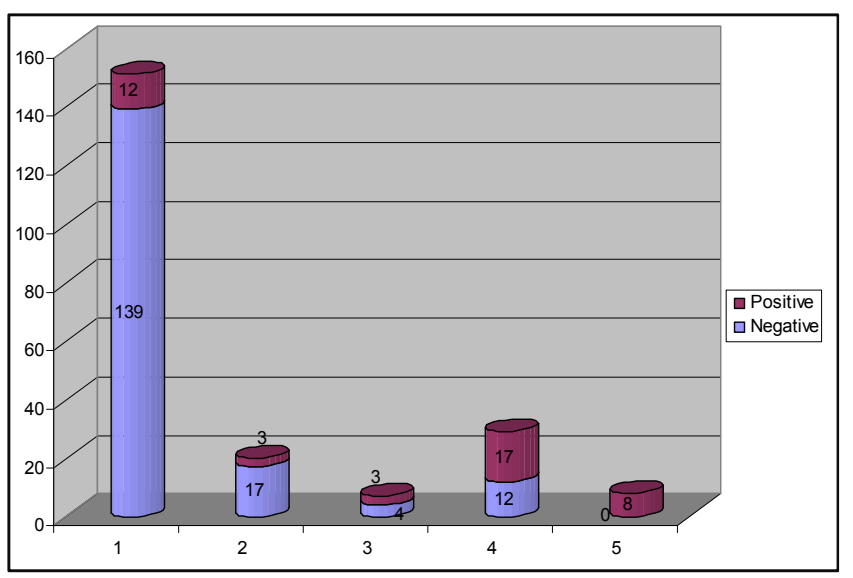

Figure 1. Comparison of cytology results with VIA results.

Figure 1 shows that $12(7.9 \%)$ of the subjects whose cytology results were normal or inflammatory were found to be VIA positive. Three (15\%) of cervicitis turned out to be VIA positive, 3 (42.9\%) of ASCUS was VIA positive, 17 $(58.6 \%)$ of low grade squamous intraepithelial lesion was positive to VIA and all women (100\%) with high grade squamous intraepithelial lesions were positive to VIA.
Table 5. Validity of VIA.

\begin{tabular}{llll}
\hline \multicolumn{2}{l}{ Cytologyresults } & & \\
\hline & Premalignant & Non-premalignant & total \\
\hline VIA & n (\%) & n (\%) & n (\%) \\
Positive & $28(13.0)$ & $15(7.0)$ & $43(20.0)$ \\
Negative & $16(7.4)$ & $156(72.6)$ & $172(80.0)$ \\
Total & $44(20.4)$ & $171(79.6)$ & $215(100)$ \\
\hline
\end{tabular}

$\chi^{2}=65.85, \mathrm{df}=1, \mathrm{p}=0.000, \mathrm{kappa}=0.553$.

True positive VIA $=28$ True negative VIA $=156$

False positive VIA $=15$ False negative VIA $=16$

Sensitivity $=$ True positive VIA / all with pre-malignant cytological lesions x $100=28 / 44$ x $100=63.6 \%$.

Specificity $=$ True Negative VIA / all with non-premalignant cytological lesions x 100=156/171 X100 =91.2\%.

Positive predictive value $=$ True positive VIA $/$ all positive VIA $\times 100=28 / 43 \times 100=0.6512 \times 100=65.1 \%$

Negative predictive value $=$ True negative VIA / all negative VIA $=156 / 172=0.9069=90.7 \%$.

Prevalence of cervical pre-malignant lesion $=$ True positive VIA + false negative VIA.

Sample size $=44 / 215=0.205=20.5 \%$.

Measure of agreement between VIA and Papanicolaou smear.

From Table 5, Kappa value $=0.553$ with p value of 0.000 . The measure of agreement, while statistically significant, is only moderately convincing (moderate agreement) based on the interpretation of kappa value as early discussed in the methodology.

\section{Discussion}

VIA is an attractive alternative to Pap smears for its ease of use, low-cost and fewer physician visits. Currently, to do a Pap smear, the doctor requires a speculum, lamp, slide, cytobrush, microscope, pathologist and a 2-week or more follow-up visit. With VIA, any trained nurse or physician able to use a speculum can do the test. Tools needed include a 
speculum, lamp, cotton swab, and acetic acid (vinegar); there is no pathologist or physician needed. And, if the test is negative, the patient can be told immediately without having to return to the doctor for results. In rural areas like ours where people travel hours for a doctors' visit, a screening method requiring fewer visits will have a much higher success rate. In this study, the performance of VIA was evaluated in comparison to Pap smear.

There was gradient agreement between VIA and Papanicolaou smear with respect to abnormal cytology findings. The association between the two tests was found to be significant $(\mathrm{p}=0.000)$. VIA was able to pick all the HGSIL $(100 \%)$ and picked $58.6 \%$ of all LGSIL.

The true sensitivity and specificity of the two screening tests could not be calculated since the researcher was not privileged to do the diagnostic investigation (cervical cone biopsy for histology) needed to compare the two tests. Sensitivity, specificity, positive and negative predictive values were calculated for VIA with respect to Pap smear results in this study. This means that Criterion Validity was used. Criterion Validity is the comparison of the result of the measure (VIA in this study) with a criterion, which is a gold standard (close to the truth, a measure with higher face validity). This is also referred to as concurrent validity. [26]

The sensitivity of VIA was $63.6 \%$, the specificity was $91.2 \%$, the positive predictive value (PPV) was $65.1 \%$ and the negative predictive value (NPV) was 90.7\%. The sensitivity of VIA in this study is lower than its specificity because of the high level of false negative VIA results $16(7.4 \%)$. The negative predictive value, which also took into consideration the false negative VIA result, was very significantly high. The false negative VIA is slightly higher than the false positive VIA $15(7.0 \%)$. It is expected that a good screening test should have a lower false negative result. However, it must be understood that two screening tests were compared, the gold standard test (Pap smear) could also have varied false negative and false positive results which could have affected the interpretation of the results of VIA in this study. The limitation of not able to do the colposcopy with cone biopsy in this study could have accounted for the higher false negative finding of VIA this study. Nevertheless, a study done in a similar tertiary Hospital in the Northern part of Nigeria where biopsy for Histology was done in 2012 reported sensitivity, specificity, PPV, NPP of $60.0 \%, 94.4 \%$, $50.0 \%, 99.4 \%$ respectively, this is quite similar to our finding. [27] Also 2015Momna K and colleagues found a similar pattern with sensitivity, specificity, PPV and NPV of 93.5\%, 95.8\%, 76.3\% and 99.0\% respectively.[28] They also had VIA sensitivity lower than its specificity even though at higher value than ours. Unlike in a study by Akinola OA et al in Lagos state teaching hospital, Nigeria, who carried out Papanicolaou smear and VIA on 185 subjects, those who had abnormal cytology results were called back for biopsies. They found sensitivity of VIA to be $100 \%$ and sensitivity of cytology was $85.7 \%$, the negative predictive value of VIA was $100 \%$ and its positive predictive value was $20 \%$. VIA detected one lesion missed by Pap smear.[29] Similarly studies done in some other Africa countries like Egypt and Kenya revealed higher level of sensitivity of VIA. [23, 30] Though, histology was not done in our study, but this is significantly high enough to validate VIA in a criterion validity. A specificity of $91.2 \%$ in a criterion validity study shows that there is an appreciable agreement between the study screening test (VIA) and the gold standard (Pap smear) in addition to the fact that VIA was able to pick $100 \%$ of HGSIL detected with Papanicolaou smear.

Screening programs were implemented in developing countries since the early 1980's, yet have failed to reduce the mortality rates. The WHO in 2002 estimated that only $5 \%$ of women in developing countries are screened appropriately. Likely reasons for failure in screening programs include lack of funding, insufficient access in rural areas where most of the population in developing countries reside, lack of awareness/education as to need for screening, and poor follow-up.[31] About 50\% of all cancers occur in developing countries, yet only $5 \%$ of resources are spent on the fight against cancer worldwide. Gaffikin in 2003 published a minimeta-analysis although a lower specificity was noted in various publications; Gaffikin published test qualities of Pap smears ranging from $11 \%$ to $99 \%$. Gaffikin concluded that VIA was comparable to Pap smears in terms of detecting HGSIL's or cancer and similar thing was found in our study.[31] She also went onto say that, although a lower specificity was noted in various publications, the authors nonetheless unanimously concluded that VIA was useful as an adjuvant or alternate to cytology.[31]

The measure of agreement between the two tests by kappa statistic was 0.553 . This is described as moderate agreement based on the grading of level of agreement as earlier discussed in the methodology. Although, this is far from perfect agreement but it is judged by the association test (chisquare) as significant. It further supports the validation of VIA as a screening test that can be used in lieu of Pap smear in the primary and secondary care settings of a low-resource settings countries like Nigeria where Papanicolaou test is not readily available.

The prevalence of pre-malignant lesion in this study was $20.5 \%$. Pre-cancerous lesions were reported to also occur in about $20 \%$ of women with HIV/AIDS in a study in Ibadan, West part Nigeria. [7] though HIV screening was not done in this study. A study in Okene, Central part of Nigeria in 2004 reported a lower figure; $13(6.5 \%)$ of 231 subjects had abnormal cytology; two were LGSIL and one was HGSIL. [32] A similar Hospital-based study in Zaria, North part of Nigeria found prevalence rate of $14 \%$ for the cervical intraepithelial neoplasia. [33] However, a lower prevalence of $1.4 \%$ was reported in a study done in 2012 in the same environment.[27]The prevalence studies around the world has shown a wide range from as low as $0.98 \%$ to as high as $15.6 \%$. [34, 35] No consistent pattern emerged in these studies both in developed and developing countries. The reasons for these variation could be many including criteria employed for diagnosis, the quality checks used, intrinsic differences in the population studied including the prevalence 
of risk factors and the numbers studied which have ranged from as few as 419 to as high as 297,849. [34, 35]

In conclusion, the level of agreement between VIA and Pap smear in this study was moderate and statistically significant. Coupled with high specificity $(91.2 \%)$ and high negative predictive value $(90.7 \%)$, it suggests that VIA may be useful to a large extent as an alternative to Pap smear for cervical cancer screening in resource limited settings. In addition VIA is simple, easy, cost effective and its result is readily available.

\section{References}

[1] Namagembe I. Cancer of the cervix and its prevention, still a public health concern. Available from www.cwru.edu/med/epidio/mphp439cervical_cancer.htm. (accessed September 5, 2015).

[2] Harris TG, Burk Rd, Palefsky JM, Massad LS, Bang JY, Anastos $\mathrm{K}$, et al. Incidence of cervical squamous intraepithelial lesions associated with HIV sero-status, CD4 cell counts, and Human Papilloma Virus test results. JAMA. 2005; 293(12):1471-1476.

[3] Bosch FX, Lorincz A, Munoz N, Meijer CJLM, Shah KV. Causal relation between Human Papilloma Virus and cervical cancer. J. Clin. Pathol. 2002; 55(4): 244-265.

[4] Amy E P, Miranda B, Lindsay E, Felicity C, Nathalie B. Ensuring access to HPVvaccine through integrated services; a reproductive health perspective. Bull. WHO.2007; 85(1): 57-63.

[5] Rengaswamy S, Atul MB, Rajamanickam R. Effective screening programmes for cervical cancer in low income developing countries. Bull. WHO.2001; 79:10.

[6] Peter OA. Knowledge and practice of cervical cancer screening among female professional health workers in a suburban district of Nigeria. Nigerian MedicalPractitioner.2006; 50(1): 19-22.

[7] Abdus-Salam AA, Ogunnorin OB, Abdus-Salam RA. HIV Seroprevalence in Patients with Carcinoma of the Cervix in Ibadan, Nigeria. Ghana Med J.2008; 42(4): 141-143.

[8] Parkin DM, Bray F, Ferlay J, Pisani P. Global cancer statistics, 2002. CA Cancer JClin.2005; 55:74-108.

[9] Parkin DM, Ferlay J, Hamdi-Cherif M, Sitas F, Thomas JO, Wabinga $\mathrm{H}$ et al. Cancer in Africa: Epidemiology and Prevention. IARC Scientific Publications.2003; 153: 268-276.

[10] Domfeh AB, Wiredu EK, Adjei AA, Ayeh-Kumi PFK, Adiku TK, Tettey Y etal. Cervical Human Papilloma Virus infection in Accra, Ghana. Ghana Med J. 2008;42(2): 71-78

[11] Thomas JO, Herrero R, Omigbodun AA, Ojemakinde K, Ajayi IO, Fawole A et al Prevalence of Papilloma Virus infection in women in Ibadan, Nigeria: a population-based study. $\mathrm{Br} J$ Cancer.2004; 90(3): 638-645.

[12] Rasha D, Jyoti V, Martha J, Ilana D, Nisha L, Jan B, et al. A strategic assessment of cervical cancer prevention and treatment services in 3 districts of Uttar Pradesh, India. Reprod Health. 2005; 2: 11.

[13] Anorlu RI. Cervical cancer; sub-saharan Africa perspective. Reprod. Health Matters. 2008; 16(32): 41-9.
[14] Nemekhee O, Young MC, Mee RK, Ji EY, Sun HJ. Association between smoking, screening, and death by cervical cancer in Korean women. Yonsei Med J. 2007; 48(2):192-2000.

[15] Ijaiya MA, Aboyeji PA, Buhari MO. Cancer of cervix in Ilorin, Nigeria. West African Journal of Medicine. 2004; 23(4): 319-322.

[16] Mandong BM, Madaki AKJ, Mannaseh AN. Malignant diseases in Jos: A follow up. Annals of African Medicine. 2004; 2(2):49-53.

[17] Gichangi P, Estambale B, Biuayo J, Rogo K, Ojwang S, Opiyo A et al. Knowledge and practice about cervical cancer and Pap smear testing among patients at Kenyatta National Hospital, Nairobi Kenya. International Journal of Gynecological Cancer. 2003; 13 (6):827-833.

[18] Robert ER (author) The Family Physician, in: Robert ER' editor Text book of Family Practice, $6^{\text {th }}$ ed. WB Saunder 2001; 13-17.

[19] Thomas LC, James HB, The family, s influence on health, in: Robert ER, editor. Textbook of Family Practice $6^{\text {th }}$ ed. WB Saunder 2001; 34-41.

[20] Katz A. Cervical cancer screening- role of Family Physician. Hong Kong practitioner 1999; 21(1): 18-22.

[21] John MAM Prevention in general practice; periodic health examination. In: John MAM (Editor) General Practice $3^{\text {rd }}$ McGraw-Hill companies; 2003: 66-76.

[22] Preventing Cervical Cancer in low resources setting. PATH'S Outlook 2000; 18 (No1): 1-6. [Updated2003 July 09]. Available from http//www.roproline.jhu.edu/3cc/3outlook (accessed 25 ${ }^{\text {th }}$ September2008).

[23] Saleh HS, Can visual inspection with acetic acid be used as an alternative to Pap smear in screening cervical cancer? Middle East Fertility Society Journal, September 2014; 19 (3): 187191.

[24] John MAM. Research and evidence-based medicine In: John MAM (Editor), General Practice $3^{\text {rd }}$ McGraw-Hill companies; 2003:131-140.

[25] Steve S. Definition category; measuring agreement. Available fromwww.children.mercy.org/stats/definition/kappa.htm. modified on $14^{\text {th }}$ july, 2008 (accessed on $29^{\text {th }}$ April 2015).

[26] Araoye OA. Data collection, In: Research Methodology with statistics for health and social sciences. Araoye OA (Editor). Ilorin; Nathadex publishers 2004; 130-159.

[27] Albert SO, Oguntayo OA, Smaila MOA, comparative study of visual inspection of the cervix using acetic acid (VIA) and papanicolaou (Pap) smear for cervical cancer screening. ecancermedicalscience. 2012; 6: 263.

[28] Momna K, Nigar J, Usman A, Visual inspection of cervix with acetic acid: a good alternative to Pap smear for cervical cancer screening in resource-limited setting, JPMA 2015; 65:192.

[29] Akinola OI, Fabamwo AO, Oshodi YA, Banjo AA, Odusanya $\mathrm{O}$, Gbadegesin A et al. Efficacy of visual inspection of cervix using acetic acid in cervical cancer screening; a comparison with cervical cytology. J obstet. Gynaecol.2007; 27(7):703705. 
[30] Huchko MJ, Sneden J, Zakaras JM, smith-McCune K, Sawaya $\mathrm{J}$, Maloba $\mathrm{M}$ et al. a Randomized Trial comparing the diagnostic accuracy of visual inspection with acetic acid to visual inspection with Lugol Iodine for cervical cancer screening in HIV infected woman. PloS 2015; 10(4): doi10.1371pone 0118568 .

[31] Gaffikin L, Lauterbach M, Blumenthal PD. Performance of Visual inspection with acetic acid for cervical cancer screening: a qualitative summary of evidence to date. Obste Gynecol Surv. 2003; 58(8):543-550.

[32] Peter FS, Natalia VM, Danielle H, Srinivas RM, David MO. The prevalence of cervical HPV and cytological
Abnormalities in association with reproductive factors of Rural and Nigerian women. Journal of Women's Health. 2008; 17(2): 279-285.

[33] Abdul MA, Shittu SO, Randawa JA, Shehu MS. The cervical smear pattern withchronic inflammatory disease. Niger $J$ ClinPract, 2009; 12(3): 289-293.

[34] Sadan O, Schejter E, Ginath S, Bachar R, Boaz M, Menczer J, et al. Premalignant lesions of the uterine cervix in a large cohort of Israeli Jewish women. Arch Gynecol Obstet. 2004; 269:188-191.

[35] Tbistle PJ, Chirenje ZM. Cervical cancer screening in a rural population of Zimbabwe. CentAfr J Med.1997; 43:246-251. 